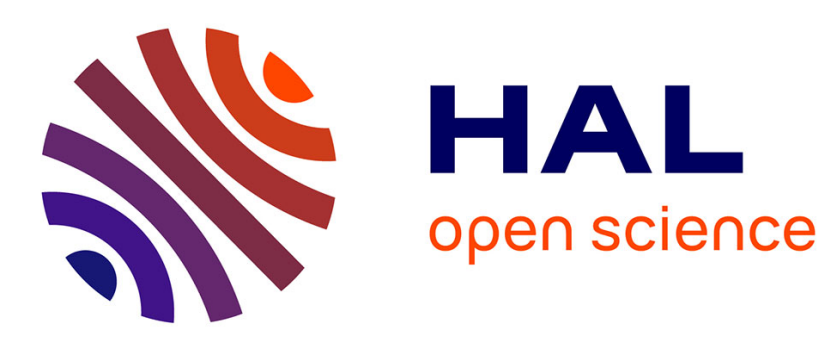

\title{
Finite-time stability and stabilization of time-delay systems
}

Emmanuel Moulay, Michel Dambrine, Nima Yeganefar, Wilfrid Perruquetti

\section{To cite this version:}

Emmanuel Moulay, Michel Dambrine, Nima Yeganefar, Wilfrid Perruquetti. Finite-time stability and stabilization of time-delay systems. Systems and Control Letters, 2008, 57 (7), pp.561-566. 10.1016/j.sysconle.2007.12.002 . inria-00344524

\section{HAL Id: inria-00344524 https://hal.inria.fr/inria-00344524}

Submitted on 19 Feb 2021

HAL is a multi-disciplinary open access archive for the deposit and dissemination of scientific research documents, whether they are published or not. The documents may come from teaching and research institutions in France or abroad, or from public or private research centers.
L'archive ouverte pluridisciplinaire HAL, est destinée au dépôt et à la diffusion de documents scientifiques de niveau recherche, publiés ou non, émanant des établissements d'enseignement et de recherche français ou étrangers, des laboratoires publics ou privés. 


\title{
Finite-time stability and stabilization of time-delay systems
}

\author{
Emmanuel Moulay *,a, Michel Dambrine ${ }^{\mathrm{b}}$, Nima Yeganefar $^{\mathrm{c}}$, Wilfrid Perruquetti ${ }^{\mathrm{c}}$ \\ a IRCCYN (UMR-CNRS 6597) \\ 1 rue de la Nö̈-BP 92 101 \\ 44321 Nantes Cedex 03, France \\ ${ }^{\mathrm{b}}$ LAMIH (UMR-CNRS 8530) - Université de Valenciennes et du Hainaut-Cambrésis \\ le Mont Houy \\ 59313 Valenciennes Cedex 9, France \\ ${ }^{\mathrm{c}} L A G I S$ (UMR-CNRS 8146)-Ecole Centrale de Lille \\ Cité Scientifique - BP 48 \\ 59651 Villeneuve d'Ascq Cedex, France
}

\begin{abstract}
Finite-time stability and stabilization of retarded type functional differential equations is developed. First, a theoretical result on finite-time stability inspired by the theory of differential equations, using Lyapunov functionals, is given. As it is not usable in practice, we show how to obtain finite-time stabilization of linear systems with delays in the input by using an extension of the Artstein's model reduction to nonlinear feedback. With this approach, we give an explicit finite-time controller for scalar linear systems and for the chain of integrators with delays in the input.
\end{abstract}

Key words: Finite-time stability, Finite-time stabilization, Time-delay systems.

\section{Introduction}

In the framework of ordinary differential equations, several studies have shown the possibility of stabilizing a system in finite-time - and not merely asymptotically — using continuous, time-invariant feedbacks. Several aspects of finite-time stability have been considered in the literature, both from a theoretical point of view with precise definitions, Lyapunov and converse Lyapunov results, regularity properties of the settling-time function [1-3], and from a practical point of view with applications in robotics [4] and from the fact that such control laws exhibit good properties of robustness and disturbance rejections [5]. Recently, finite-time stabilization for triangular control systems described by retarded functional differential equations has been studied in [6] by using the back-stepping technique.

First, in this paper, we show how to obtain a theoretical sufficient condition of finite-time stability for general

\footnotetext{
* (33) +2 40376927

Email addresses: Emmanuel.Moulay@irccyn.ec-nantes.fr (Emmanuel Moulay), michel.dambrine@univ-valenciennes.fr (Michel Dambrine), Nima.Yeganefar@ec-lille.fr (Nima Yeganefar), wilfrid.perruquetti@ec-lille.fr (Wilfrid Perruquetti).
}

retarded functional differential equations by using the method of Lyapunov-Krasovskii functionals. This result is inspired by the results on finite-time stability of ordinary differential equations (see [1-3]) and can be easily extended to the neutral-type cases. Unfortunately, this theoretical result is not so usable in practice for studying the finitetime stabilization problem. This is due to the fact that it is really difficult to find a Lyapunov functional satisfying the assumptions of our theoretical result. Only a simple example is given; the reader may understand that this result is a only theoretical one. This is the reason why we develop a more practical method. So, we will demonstrate how an extension of the theory of the Artstein's reducing transformations defined in [7] can be used to solve the finite-time stabilization problem of linear delayed control systems. This kind of models are generally used to describe systems with delay on the transmission lines as in the teleoperation or remote systems. The presented method is then used to give a finite-time feedback control for scalar linear systems and for the chain of integrator with delays in the input.

The paper is organized as follows. In section 2, we will provide some notations and definitions useful all along the paper. A sufficient condition for finite-time stability of retarded functional differential equations will be addressed in 
section 3. In section 4, an extension of the Artstein models reduction will be given. The reduction enables us to employ the literature on ordinary systems, and to analyze the finite-time stabilization of linear systems with delays on the input variables with an example. Finally, the conclusions of the paper are given in section 5 .

\section{Notations and definitions}

The upper right Dini derivative of a function $f:[a, b] \rightarrow$ $\mathbb{R}$ is the function $D^{+} f:[a, b] \rightarrow \overline{\mathbb{R}}$ defined by

$$
D^{+} f(x)=\limsup _{h \rightarrow 0^{+}} \frac{f(x+h)-f(x)}{h} .
$$

Let $a>0$, a continuous function $g:[0, a] \rightarrow[0,+\infty[$ belongs to class $\mathcal{K}$ if it is strictly increasing and $g(0)=0$. Let $A$ be a matrix, $x \in \mathbb{R}^{n}$ and $f$ a real function, $\||A|\|$ denotes a matrix norm,

$$
\|f\|_{\infty}=\sup _{x \geq 0}|f(x)|
$$

the infinity norm and $\|x\|_{n}$ the Euclidean norm in $\mathbb{R}^{n}$. The spectrum of a matrix $A$ is denoted by $\sigma(A)$.

Throughout this paper, we use the following notations: $C_{n}^{h}$ is the space of continuous functions $\phi:[-h, 0] \rightarrow \mathbb{R}^{n}$ with $h \geq 0$, and

with

$$
\mathbf{C}_{\epsilon}=\left\{\phi \in C_{n}^{h}:\|\phi\|_{C_{n}^{h}}<\epsilon\right\}
$$

$$
\|\phi\|_{C_{n}^{h}}=\sup _{-h \leq s \leq 0}\|\phi(s)\|_{n} .
$$

We are interested by retarded functional differential equations of the form

$$
\dot{x}(t)=\mathbf{f}\left(x_{t}\right), \quad t \geq 0,
$$

where $x_{t}:[-h, 0] \rightarrow \mathbb{R}^{n}$ is given by $x_{t}(s)=x(t+s)$ and $\dot{x}_{t}(s)=\dot{x}(t+s)$ for every $s \in[-h, 0]$. $\dot{x}(t)$ denotes the right hand derivative of $x(t), \mathbf{f}: C_{n}^{h} \rightarrow \mathbb{R}^{n}$ is a continuous functional such that $\mathbf{f}(\mathbf{0})=0$. In the following, we suppose that the system (1) possesses uniqueness of solutions in forward time.

As usual, $x(t)$ is called a solution of the system (1) with initial condition $\phi$ at the origin if $x(t)$ is defined on $[-h, b)$ with $b \in \overline{\mathbb{R}}$ such that:

(i) $x_{0} \equiv \phi$,

(ii) $x(t)$ is continuous on $[0, b)$,

(iii) $x(t)$ satisfies the equation (1) for all $t \in[0, b)$.

$x(t)$ is denoted by $x(t, \phi)$ and $x_{t}$ by $x_{t}(\phi)$.

The system (1) is said to be:

(i) stable if for any $\epsilon>0$ there exists $\delta(\epsilon)>0$, such that $\phi \in \mathbf{C}_{\delta(\epsilon)}$ implies that

(a) $x(t, \phi)$ is defined for all $t \geq 0$,

(b) $\|x(t, \phi)\|_{n}<\epsilon$ for all $t \geq 0$,

(ii) asymptotically stable if it is stable and there exists $\delta$ such that $\phi \in \mathbf{C}_{\delta}$ implies $\lim _{t \rightarrow+\infty} x(t, \phi)=0$.

Definition 1 The system (1) is finite-time stable if: (i) the system (1) is stable for the system (1),

(ii) there exists $\delta>0$ such that, for any $\phi \in \mathbf{C}_{\delta}$, there exists $0 \leq T(\phi)<+\infty$ for which $x(t, \phi)=0$ for all $t \geq T(\phi)$.

$$
T_{0}(\phi)=\inf \{T(\phi) \geq 0: x(t, \phi)=0 \quad \forall t \geq T(\phi)\}
$$

is a functional called the settling time of the system (1).

Remark 2 The finite-time stability implies that the system (1) cannot be Lipschitz at the origin. Indeed, if the system (1) is finite-time stable, there is no uniqueness of solutions in backward time at the origin due to the fact that all solutions reach the origin. This motivates the assumption on the continuity of the system (1) with uniqueness of solutions in forward time. It implies the existence of a global semi-flow and the continuous dependence on the initial data (see [8, Chapitre 2]).

\section{Preliminary results on finite-time stability}

The first lemma is called the Comparison Lemma. Its proof and more general versions can be found in [9, Section 5.2] and [10, Section 4].

Lemma 3 (Comparison Lemma) Let $J$ be a segment of $\mathbb{R}$, if the scalar differential equation

$$
\dot{x}=f(x), \quad x \in J
$$

has the global semi-flow $\Phi: \mathbb{R}_{>0} \times J \rightarrow \mathbb{R}$, where $f: J \rightarrow \mathbb{R}$ is continuous, and if $g:[a, b) \rightarrow J$ (b could be infinity) is a continuous function such that

$$
D^{+} g(t) \leq f(g(t)), \quad t \in[a, b),
$$

then $g(t) \leq \Phi(t, g(a))$ for all $t \in[a, b)$.

We denote by

$$
\begin{aligned}
\dot{\mathbf{V}}\left(x_{t}\right) & =\limsup _{h \rightarrow 0^{+}} \frac{\mathbf{V}\left(x_{t+h}\right)-\mathbf{V}\left(x_{t}\right)}{h} \\
& =D^{+} \mathbf{V}\left(x_{t}\right) .
\end{aligned}
$$

Proposition 4 Consider the system (1) with uniqueness of solutions in forward time. If there exists $\delta>0$ and a continuous functional $\mathbf{V}: \mathbf{C}_{\delta} \rightarrow \mathbb{R}_{\geq 0}, \epsilon>0$, two functions $\alpha, r$ of class $\mathcal{K}$ such that $\dot{z}=r(z)$ has a flow and for all $\phi \in \mathbf{C}_{\delta}$,

(i) $\alpha\left(\|\phi(0)\|_{n}\right) \leq \mathbf{V}(\phi)$,

(ii) $\dot{\mathbf{V}}(\phi) \leq-r(\mathbf{V}(\phi))$ with $\int_{0}^{\epsilon} \frac{d z}{r(z)}<+\infty$,

then the system (1) is finite-time stable with a settling time satisfying the inequality

$$
T_{0}(\phi) \leq \int_{0}^{\mathbf{V}(\phi)} \frac{d z}{r(z)} .
$$

Proof. Let $\mathbf{V}: \mathbf{C}_{\delta} \rightarrow \mathbb{R}_{\geq 0}$ be a Lyapunov functional for the system (1). The Lyapunov-Krasovskii theorem (see [8, $\S 5.3]$ ) ensures that the system (1) is asymptotically stable. Let $x(t, \phi)$ be a solution of (1) which tends to the origin 
with the settling time $0 \leq T_{0}(\phi) \leq+\infty$. It remains to prove that $T_{0}(\phi)<+\infty$. Let us consider the system

$$
\dot{y}=-r(y), \quad y \geq 0,
$$

with the flow $\Phi(t, y)$ for $y \geq 0$. For all $t \geq 0$ and all $\phi \in \mathbf{C}_{\delta}$,

$$
\dot{\mathbf{V}}\left(x_{t}(\phi)\right) \leq-r\left(\mathbf{V}\left(x_{t}(\phi)\right)\right) \text {. }
$$

Now, if we apply the Comparison Lemma 3, we could deduce that

$$
\mathbf{V}\left(x_{t}(\phi)\right) \leq \Phi(t, \mathbf{V}(\phi)), \quad t \geq 0, \phi \in \mathbf{C}_{\delta}
$$

From [2], we know that

$$
\Phi(t, \mathbf{V}(\phi))=0 \quad \text { for } \quad t \geq \int_{0}^{\mathbf{V}(\phi)} \frac{d z}{r(z)} .
$$

With the positive definiteness of $\mathbf{V}$, we could conclude that, for all $t \geq 0$ and all $\phi \in \mathbf{C}_{\delta}$,

$$
x(t, \phi)=0 \quad \text { for } \quad t \geq \int_{0}^{\mathbf{V}(\phi)} \frac{d z}{r(z)} .
$$

Thus, the system (1) is finite-time stable with the settling time

$$
T_{0}(\phi) \leq \int_{0}^{\mathbf{V}(\phi)} \frac{d z}{r(z)}<+\infty .
$$

Thus the system (1) is finite-time stable. It is worth noticing that $T_{0}$ is actually continuous at the origin.

Let us consider a simple example of a finite-time stable retarded system.

Example 5 Let $0<\alpha<1$ and consider the delayed system

$$
\dot{x}(t)=-|x(t)|^{\alpha} \operatorname{sgn}(x(t))\left(1+x(t-h)^{2}\right) .
$$

The solutions of such a system depend continuously on the initial data. Indeed, the functional is Lipschitz everywhere in $x(t-h)$ and Lipschitz outside the origin and continuous everywhere in $x(t)$. We know that the Lipschitz property of the right hand side of the system implies the uniqueness solutions. As the only solution starting from the origin of the system is the zero solution $x(t) \equiv 0$, the system possesses uniqueness of solutions in forward time. Let

$$
\mathbf{V}\left(x_{t}\right)=\frac{x(t)^{2}}{2}
$$

we have

$$
\begin{aligned}
\dot{\mathbf{V}}\left(x_{t}\right) & =-|x(t)|^{1+\alpha}\left(1+x(t-h)^{2}\right) \\
& \leq-|x(t)|^{1+\alpha}=-2^{\frac{1+\alpha}{2}} \mathbf{V}\left(x_{t}\right)^{\frac{1+\alpha}{2}} .
\end{aligned}
$$

So the system is finite-time stable under the settling time

$$
T_{0}\left(x_{t}\right) \leq \frac{|x(t)|^{1-\alpha}}{1-\alpha} .
$$

Figure 1 shows the evolution of $x(t)$ for $\alpha=h=0.5$ and $x_{0} \equiv 0$.

Remark 6 Let us consider the system

$$
\dot{x}(t)=f\left(x(t), x\left(t-h_{1}\right), \ldots, x\left(t-h_{k}\right)\right)
$$

where $h_{1}, \ldots, h_{k}$ are positive numbers and

$$
f: \mathbb{R}^{n} \times \ldots \times \mathbb{R}^{n} \rightarrow \mathbb{R}^{n}
$$

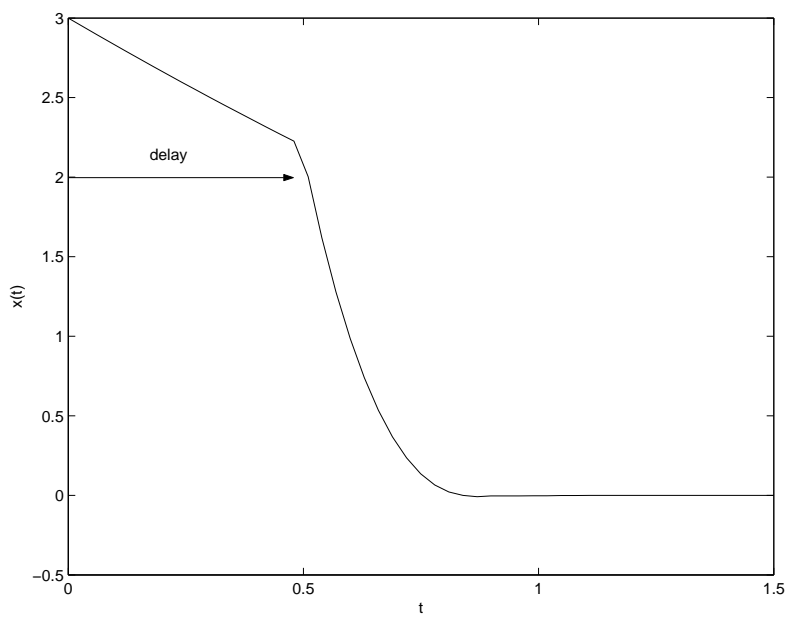

Fig. 1. A finite time stable delayed system with $h=0.5$

a non zero continuous function such that the origin is the only point of the domain of $f$ satisfying $f(0, \ldots, 0)=0$. Then the system can not be finite-time stable. Indeed, suppose that the system is finite-time stable. Let $x(t)$ be a solution satisfying $x(t)=0$ for all $t \geq T$. It implies that $\dot{x}(t)=0$ for all $t \geq T$. With the assumption on $f$, we deduce that $x(t)=0$ for all $t \geq T-\max \left(h_{1}, \ldots, h_{k}\right)$. Step by step, we conclude that $x(t)=0$ for all $t \geq 0$, then $x(t) \equiv 0$ and $f \equiv 0$. This leads to a contradiction.

The previous remark implies that there is no way to solve the finite-time stabilization problem for the class of discrete delayed systems whose closed-loop system is defined by (2). In particular, the use of a discrete delayed feedback control is inadequate to solve the finite-time stabilization problem. In order to give a constructive method for the problem of finite-time stabilization of a class of linear systems, we will use the Artstein's reduction-type scheme which leads to the use of a feedback control with distributed-delays.

\section{Extension of the Artstein's transformation: application to the finite-time stabilization problem}

Let us consider the system

$$
\dot{x}(t)=A x(t)+\sum_{i=0}^{k} B_{i} u\left(t-h_{i}\right), \quad t \geq 0
$$

where $x(t) \in \mathbb{R}^{n}, u(t) \in \mathbb{R}^{m}, A$ is a $n \times n$ matrix, the matrices $B_{i}$ are $n \times m, h_{i}$ are positive constants. The control system (3) is finite-time stabilizable if there exists a feedback control law $u$ which is time and state dependent such that:

(i) $u(0)=0$,

(ii) the closed-loop system

$$
\dot{x}(t)=A x(t)+\sum_{i=0}^{k} B_{i} u\left(t-h_{i}\right), \quad x \in \mathbb{R}^{n} .
$$

is a finite-time stable.

In order to simplify the notation, we denote $u(t)$ the feedback control even if it is state dependant, in order to 
easily calculate $u\left(t-h_{i}\right)$. Such kind of systems are generally used to represent the time delay introduced by transmission lines as, for example, in teleoperation or network controlled systems (see [11]). By using the Leibniz's formula for differentiating integrals depending on parameters, we deduce that if

$$
y(t)=x(t)+\sum_{i=0}^{k} \mathbf{L}_{\left(A, B_{i}\right)}^{h_{i}} u_{t}
$$

where $u_{t}:[-h, 0] \rightarrow \mathbb{R}^{m}$ is given by $u_{t}(s)=u(t+s)$ for every $s \in[-h, 0]$ and

$$
\mathbf{L}_{\left(A, B_{i}\right)}^{h} f=\int_{-h}^{0} e^{A(-h-s)} B_{i} f(s) d s
$$

then

$$
\dot{y}(t)=A y(t)+B u(t)
$$

with

$$
B=\sum_{i=0}^{k} e^{-A h_{i}} B_{i} .
$$

Now, we may give an extension of the Artstein's model reduction given in [7, Theorem 6.1] and [12] to nonlinear feedback.

Theorem 7 If the system (4) is stabilizable (respectively finite-time stabilizable) by a feedback control

$$
u(t)=k(t) f(y(t))
$$

with $k(t)$ bounded and $f: \mathbb{R}^{n} \rightarrow \mathbb{R}^{m}$ continuous such that $f(0)=0$ and there exists a function $\alpha$ of class $\mathcal{K}$ such that

$$
\|f(x)\|_{m} \leq \alpha\left(\|x\|_{n}\right),
$$

then the system (3) is stabilizable (respectively finite-time stabilizable) by the feedback control

$$
u(t)=k(t) f\left(x(t)+\sum_{i=0}^{k} \mathbf{L}_{\left(A, B_{i}\right)}^{h_{i}} u_{t}\right) .
$$

Proof. The fact that the integral equation (5) admits a continuous solution is left to the reader. Suppose that the system (4) is stabilizable by a feedback control

$$
u(t)=k(t) f(y(t))
$$

with $k(t)$ bounded and $f$ continuous such that $f(0)=0$. First, we notice that

$$
\begin{aligned}
\left\|f \circ y_{t}\right\|_{C_{m}^{h_{i}}} & =\max _{-h_{i} \leq s \leq 0}\|f(y(t+s))\|_{m}, \\
& \leq \max _{-h_{i} \leq s \leq 0} \alpha\left(\|y(t+s)\|_{n}\right) \text { by assumption, } \\
& \leq \alpha\left(\max _{-h_{i} \leq s \leq 0}\|y(t+s)\|_{n}\right) \text { since } \alpha \in \mathcal{K} \\
& \leq \alpha\left(\left\|y_{t}\right\|_{C_{n}^{h_{i}}}\right) .
\end{aligned}
$$

So, we have

$$
\begin{aligned}
& \|x(t)\| \leq\|y(t)\|+\sum_{i=0}^{k}\left\|\int_{-h_{i}}^{0} e^{A\left(-h_{i}-s\right)} B_{i} u(t+s) d s\right\| \\
& \leq\|y(t)\|+\sum_{i=0}^{k} h_{i}\left\|\left.e^{A \cdot \|}\right|_{C_{n}^{h_{i}}}\right\| B_{i}\|\|\left\|_{t}\right\|_{C_{m}^{h_{i}}} \\
& \leq\|y(t)\|+\sum_{i=0}^{k} h_{i}\left\|e^{A \cdot \|}\right\|_{C_{n}^{h_{i}}}\left\|\mid B_{i}\right\|\|\| k\left\|_{\infty}\right\| f \circ y_{t} \|_{C_{m}^{h_{i}}} \\
& \leq\|y(t)\|+\sum_{i=0}^{k} h_{i}\left\|e^{A \cdot \|}\right\|_{C_{n}^{h_{i}}}\left\|\mid B_{i}\right\|\|\| k \|_{\infty} \alpha\left(\left\|y_{t}\right\|_{C_{n}^{h_{i}}}\right) .
\end{aligned}
$$

The characterization of the asymptotic stability given for example in [13, Lemma 4.5] leads to the fact that if $y(t)$ tends asymptotically to the origin then $x(t)$ tends also. This proof is also true for finite-time stability.

Remark 8 The realization-implementation of the feedback control (5) is a challenge. To do that, it would be useful to use some results given in [14] for the Smith predictor.

Here we want to develop the finite-time stabilization of scalar time delayed systems. Consider the system

$$
\dot{y}=a y+b u, \quad y, u \in \mathbb{R}
$$

with $b \neq 0$.

Proposition 9 Let $a, b_{i} \in \mathbb{R}$ for $0 \leq i \leq k$, the system

$$
\dot{x}(t)=a x(t)+\sum_{i=0}^{k} b_{i} u\left(t-h_{i}\right), \quad t \in \mathbb{R}
$$

is finite-time stabilizable under the feedback control

$$
u(t)=\frac{-1}{b}\left[a y(t)+|(y(t))|^{\alpha} \operatorname{sgn}(y(t))\right]
$$

where $0<\alpha<1$,

$$
y(t)=x(t)+\sum_{i=0}^{k} \mathbf{L}_{\left(a, b_{i}\right)}^{h_{i}} u_{t}
$$

and

$$
b=\sum_{i=0}^{k} b_{i} e^{-a h_{i}}
$$

Moreover, the settling time satisfies

$$
T_{0}\left(x_{t}\right) \leq \frac{|y(0)|^{1-\alpha}}{1-\alpha}+\max _{0 \leq i \leq k} h_{i} .
$$

Proof. System (6) is finite-time stabilizable by the feedback control

$$
u=\frac{-a y-|y|^{\alpha} \operatorname{sgn}(y)}{b}
$$

with $0<\alpha<1$ and the settling time function is given by

$$
T(y(t))=\frac{|y(0)|^{1-\alpha}}{1-\alpha}
$$

(see [2]). By using Theorem 7 with

$$
b=\sum_{i=0}^{k} b_{i} e^{-a h_{i}}
$$

we have the first part of the result. 
Now, we have to prove is that

$$
T_{0}\left(x_{t}\right) \leq \frac{|y(0)|^{1-\alpha}}{1-\alpha}+\max _{0 \leq i \leq k} h_{i} .
$$

Equation (8) leads to the fact that

$$
y(t)=x(t)+\sum_{i=0}^{k} \mathbf{L}_{\left(a, b_{i}\right)}^{h_{i}} u_{t}
$$

reaches the origin in a finite amount of time

so

$$
T(y(t))=\frac{|y(0)|^{1-\alpha}}{1-\alpha}
$$

$$
x(t)=-\sum_{i=0}^{k} \mathbf{L}_{\left(a, b_{i}\right)}^{h_{i}} u_{t}
$$

for all $t \geq \frac{|y(0)|^{1-\alpha}}{1-\alpha}$. In the same time

$$
u(t)=\frac{-1}{b}\left[a y(t)+|(y(t))|^{\alpha} \operatorname{sgn}(y(t))\right]=0
$$

for all $t \geq \frac{|y(0)|^{1-\alpha}}{1-\alpha}$. Then,

$$
\mathbf{L}_{\left(a, b_{i}\right)}^{h_{i}} u_{t}=\int_{-h_{i}}^{0} e^{a\left(-h_{i}-s\right)} b_{i} u(t+s) d s
$$

becomes zero for all $t \geq \frac{|y(0)|^{1-\alpha}}{1-\alpha}+h_{i}$. As it is true for all $0 \leq i \leq k$, we deduce that

$$
T_{0}\left(x_{t}\right) \leq \frac{|y(0)|^{1-\alpha}}{1-\alpha}+\max _{0 \leq i \leq k} h_{i} .
$$

Let us give an illustrative example of Proposition 9.

Example 10 By using Proposition 9, we know that the system

$$
\dot{x}(t)=x(t)+u(t-h)
$$

is finite-time stabilizable under the feedback control

$$
u(t)=-y(t)-|(y(t))|^{\frac{1}{2}} \operatorname{sgn}(y(t))
$$

with

$$
y(t)=x(t)+\mathbf{L}_{(1,1)}^{h} u_{t} .
$$

This leads to the simulation on figure 2.

Remark 11 We may notice that the feedback controls in Theorem 7 and Proposition 9 involve storing the past control input history and computing an integral at every instant. As it has been mentioned in the introduction, these feedback controls are with distributed-delays.

Finally, we consider the problem of finite time stabilization of the chain of integrator with delay in the input given by

$$
\left\{\begin{array}{l}
\dot{x}_{1}(t)=x_{2}(t) \\
\vdots \\
\dot{x}_{n-1}(t)=x_{n}(t) \\
\dot{x}_{n}(t)=u(t-h)
\end{array}\right.
$$

and denoted in short by

$$
\dot{x}(t)=\breve{A} x(t)+\breve{B} u(t-h)
$$

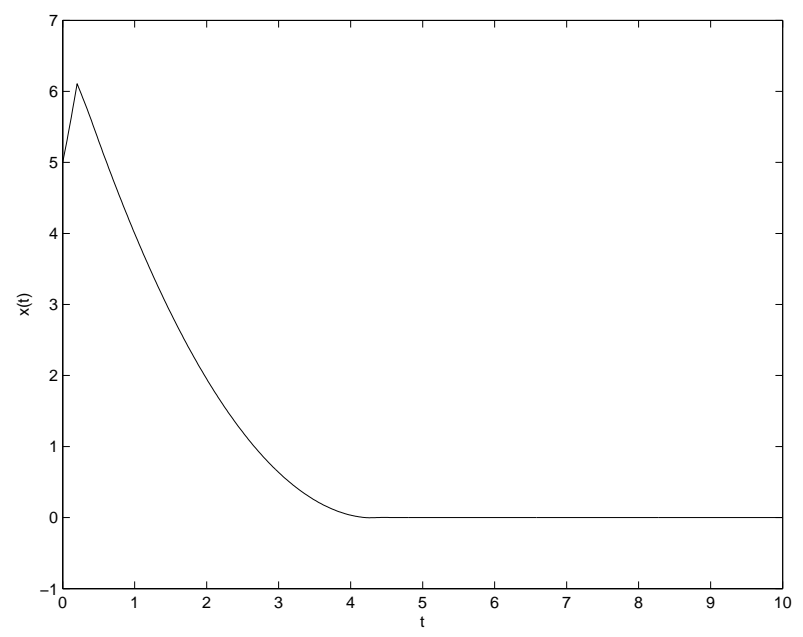

Fig. 2. Simulation with $h=0.2, x(0)=0$ and $u(t)=0$ for $t<0$ with

$$
\breve{A}=\left[\begin{array}{ccccc}
0 & 1 & 0 & \cdots & 0 \\
\vdots & \ddots & \ddots & \ddots & \vdots \\
\vdots & & \ddots & 1 & \vdots \\
0 & \cdots & \cdots & 0 & 0
\end{array}\right], \quad \breve{B}=\left(\begin{array}{c}
0 \\
\vdots \\
0 \\
1
\end{array}\right)
$$

Proposition 12 Let $k=\left(k_{1}, \ldots, k_{n}\right) \in \mathbb{R}^{n}$ such that the polynomial $s^{n}+k_{n} s^{n-1}+\ldots+k_{2} s+k_{1}$ is Hurwitz, there exists $\epsilon \in(0,1)$ such that for all $\alpha \in(1-\epsilon, 1)$, the system (9) is globally finite time stabilizable under the continuous feedback control

$$
u_{\alpha}(t)=-k_{1}\left\lfloor y_{1}(t)\right\rceil^{\alpha_{1}}-\ldots-k_{n}\left\lfloor y_{n}(t)\right\rceil^{\alpha_{n}}
$$

where

$$
\begin{aligned}
& \lfloor x\rceil^{\alpha}=|x|^{\alpha} \operatorname{sgn}(x), \quad x \in \mathbb{R} \\
& y(t)=x(t)+\mathbf{L}_{(\breve{A}, B)}^{h} u_{t}, \quad B=e^{-A h} \breve{B}
\end{aligned}
$$

and $\alpha_{1}, \ldots, \alpha_{n}$ satisfy

$$
\left\{\begin{array}{l}
\alpha_{i-1}=\frac{\alpha_{i} \alpha_{i+1}}{2 \alpha_{i+1}-\alpha_{i}}, \quad 2 \leq i \leq n \\
\alpha_{n}=\alpha \\
\alpha_{n+1}=1
\end{array}\right.
$$

The proof is a direct consequence of Theorem 7 and $[15$, Proposition 8.1].

\section{Conclusions}

Here, we are interested in the finite-time stability of functional differential equations and the finite-time stabilization problem of linear systems with delays in the input. A general theoretical result involving the Lyapunov functional gives a general sufficient condition for the finite-time stability of RFDEs and an example of a finite-time stable delayed system is addressed. Nevertheless, this result is not practical and cannot be used to stabilize in finite time a 
large class of linear systems. This is the reason why we expand the Artstein's model reduction to nonlinear feedback, in order to use a finite-time controller for a linear system with delays in the input. This lead to the finite-time stabilization of linear scalar systems and of the chain of integrator under a distributed delayed feedback control.

\section{Acknowledgment}

The authors would like to thank Jean-Pierre Richard and Lotfi Belkoura for many useful conversations on the subject of this paper.

\section{References}

[1] S. P. Bhat, D. S. Bernstein, Finite time stability of continuous autonomous systems, SIAM J. Control Optim. 38 (3) (2000) $751-766$.

[2] V. T. Haimo, Finite time controllers, SIAM J. Control Optim. 24 (4) (1986) 760-770.

[3] E. Moulay, W. Perruquetti, Finite time stability and stabilization of a class of continuous systems, J. Math. Anal. Appl. 323 (2) (2006) 1430-1443.

[4] Y. Hong, Y. Xu, J. Huang, Finite-time control for robot manipulators, Systems Control Lett. 46 (2002) 243-253.

[5] S. P. Bhat, D. S. Bernstein, Continuous finite-time stabilization of the translational and rotational double integrator, IEEE Trans. Automat. Control 43 (5) (1998) 678-682.

[6] I. Karafyllis, Finite-time global stabilization using time-varying distributed delay feedback, SIAM J. Control Optim. 45 (1) (2006) 320-342.

[7] Z. Artstein, Linear systems with delayed controls: A reduction, IEEE Trans. Automat. Control 27 (4) (1982) 869-879.

[8] J. K. Hale, S. M. V. Lunel, Introduction to Functional Differential Equations, Vol. 99, Springer-Verlag, 1993.

[9] A. Kartsatos, Advanced Ordinary Differential Equations, Mariner Publishing, Tampa, 1980.

[10] T. Yoshizawa, Stability Theory by Liapunov's Second Method, The Mathematical Society of Japon, 1966.

[11] L. G. Bushnell, Networks and control, IEEE Control Systems Magazine 22 (1) (2001) 22-99.

[12] W. H. Kwon, A. E. Pearson, Feedback stabilization of linear systems with delayed control, IEEE Trans. Automat. Control AC-25 (2) (1980) 266-269.

[13] H. K. Khalil, Nonlinear Systems, Third Edition, Prentice-Hall, Upper Saddle River, 2002.

[14] R. Wright, C. Kravaris, Nonlinear decoupling control in the presence of sensor and actuator deadtimes, Chem. eng. sci. 58 (14) (2003) 3243-3256.

[15] S. P. Bhat, D. S. Bernstein, Geometric homogeneity with applications to finite-time stability, Math. Control Signals Systems 17 (2005) 101-127. 